\title{
Partial cystectomy: Is it a reliable option for the treatment of bladder leiomyosarcoma?
}

\author{
Yun-fei Xu, MD; Guang-chun Wang, Mch(Urol); Jun-hua Zheng, MD, PhD; Bo Peng, MD
}

\begin{abstract}
Bladder leiomyosarcoma is a unique mesenchymal tumour, accounting for less than $0.5 \%$ of all primary bladder malignancies. Bladder leiomyosarcoma used to be treated with radical surgery in either old or young patients, often resulting in significant impact on the patients's quality of life after surgery. We report on a case of bladder leiomyosarcoma in a 31-year-old female who was treated with partial cystectomy. Fortunately, no tumour metastasis or relapse was observed during the 7-year follow-up period and the patient now has a good quality of life. We found that partial cystectomy may be an acceptable option to treat bladder leiomyosarcoma in the low MSKCC (Memorial Sloan-Kettering Cancer Center) stage.
\end{abstract}

Cite as: Can Urol Assoc J 2011;5(1):el 1-e13; D01:10.5489/cuaj.10031

\section{Introduction}

Non-urothelial neoplasms of the bladder account for less than $5 \%$ of all bladder tumours. Sarcoma constitutes the most usual mesenchymal malignancy of the bladder, and leiomyosarcoma is the most common type of sarcoma, accounting for less than $0.5 \%$ of bladder malignancies. ${ }^{1}$ The distinctive gross and macroscopic features of this disease were first described by Bergman and colleagues in 1950. ${ }^{2}$ Bladder leiomyosarcoma has always been considered a highly aggressive tumour, and has been treated with radical cystectomy, often resulting in significant influences on the quality of the patient's postoperative life. ${ }^{3}$ Recently, partial surgery, due to its preservation functions and lack of aggressiveness, has been considered a reliable option to treat various tumours. This article reports a case of bladder leiomyosarcoma treated with partial cystectomy, where no tumour metastasis or relapse was observed during a 7-year follow-up period. The patient now experiences a good quality of life.

\section{Case report}

A 31-year-old woman consulted our clinic because of frequent micturition and odynuria for 2 months. She was primarily diagnosed as having a urinary tract infection refractory to oral antibiotics. A week later, she was hospitalized because a B-type ultrasonography revealed a solid mass of $4 \mathrm{~cm}$ in the left wall of the bladder. Subsequent pelvic computed tomography $(\mathrm{CT})$ and magnetic resonance imaging (MRI) revealed an oval low-density tumour of about $4.0 \mathrm{~cm} \times 3.5 \mathrm{~cm}$ in the left lower wall of the bladder, with a clear margin in the mesentery and abdominal wall. No enlarged lymph node was found in the pelvic CT (Fig. 1). A cystoscopic biopsy of the bladder tumour initially revealed leiomyosarcoma pathologically. In addition, no nodal or metastatic disease was seen on abdominal CT, bone scanning or chest radiography.

Partial cystectomy was performed on December 3, 2002. The size of the bladder tumour retrieved through the cystectomy was $4.0 \mathrm{~cm} \times 3.5 \mathrm{~cm} \times 3 \mathrm{~cm}$. Grossly, the tumour located at the left wall of the bladder and above the left ureteral orifice was smooth and encapsulated, but it infiltrated the muscular layer. Microscopy revealed elongated cells with blunt-end nuclei arranged in interlacing cords and with large prominent anaplastic features and pyknotic nuclei (Fig. 2) (Fig. 3). Postoperative pathology confirmed the diagnosis of bladder leiomyosarcoma with low potential malignancy in T1 and MSKCC (Memorial Sloan-Kettering Cancer Center soft-tissue sarcoma staging system) stage 1 . Complete resection with negative surgical margins was achieved. No postoperative radiotherapy or chemotherapy was performed in this patient. The patient was followed up by chest X-ray, abdominal pelvic CT scan and cystoscopic biopsy in postoperative month 3, 6 and 12, and then annually for the following 6 years. At present, her bladder capacity is about $320 \mathrm{~mL}$, the frequency of nycturia is 0 to 2 times per night and her urination is well-controlled. She is free of tumour metastasis and living a satisfactory life (Fig. 4). 


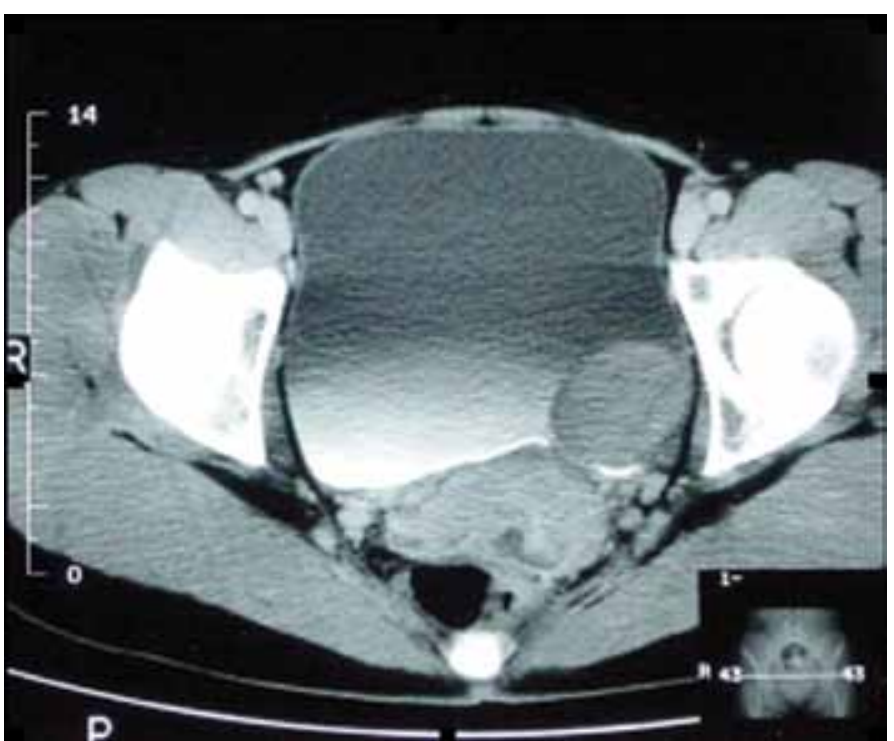

Fig. 1. An oval tumour about $4.0 \mathrm{~cm} \times 3.5 \mathrm{~cm}$ in the left lower wall of the bladder; it has a clear margin with the mesentery and abdominal wall.

\section{Discussion}

Bladder leiomyosarcoma is a rare tumour. Although it is the most common type of bladder sarcoma, very little is known about its origin, clinicopathologic presentation, treatment and prognosis. What is known about bladder leiomyosarcoma is that it has a grim prognosis.

The mean onset age of bladder leiomyosarcoma is 52 years, with a higher incidence in females at a reproductive age, which suggests that hormones play a role in the diagnosis. There is strong evidence that the incidence of bladder leiomyosarcoma is on the increase in patients receiving local pelvic radiotherapy or systemic chemotherapy, especially in cyclophosphamide therapy. ${ }^{4}$ Smoking is not a risk factor of bladder leiomyosarcoma. The predilection sites of bladder leiomyosarcoma are the vertex of bladder $(50 \%)$ and the

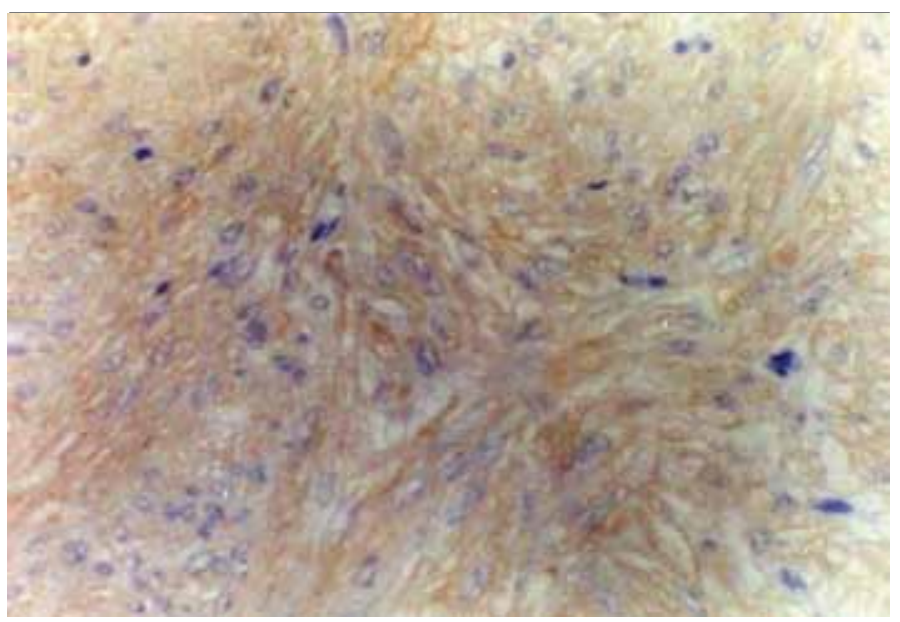

Fig. 3. Immunohistochemistry: SMA-positive tumour cells presented brown, $\times 400$.

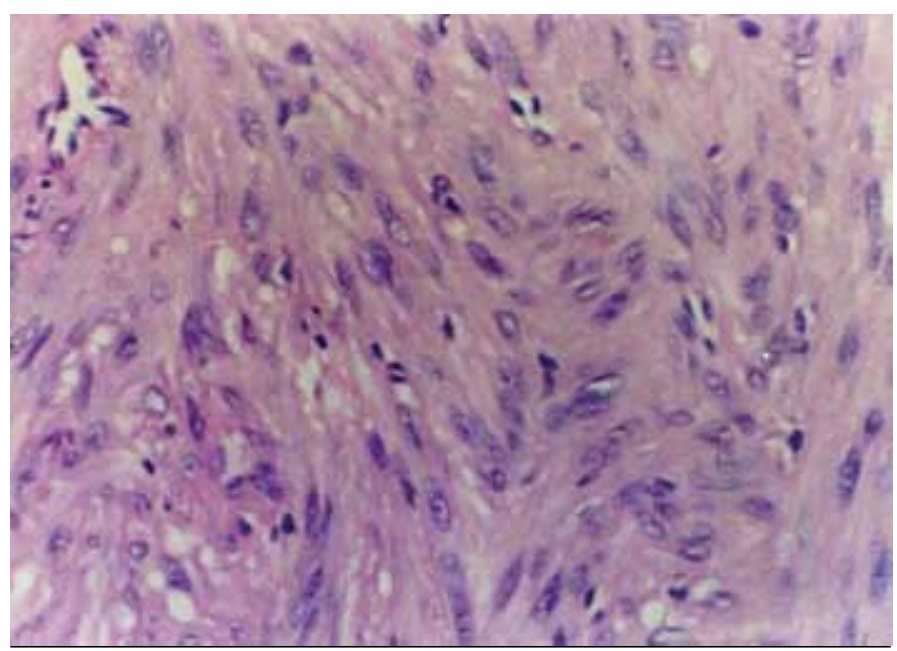

Fig. 2. Tumour cells presented pleomorphic, atypical hyperplasia and mitotic figures in some regions, hematoxylin and eosin $\times 400$.

lateral wall of the bladder (25\%). It is relatively rare in the trigone of bladder. ${ }^{1}$ The clinical presentation relates mostly to out-flow obstruction and irritative symptomatology. Most of these tumours are located in the submucosa, and few are in the muscularis propria or extended outside the wall or are multifocal. They usually present as well-circumscribed grayish firm nodules. ${ }^{5}$

Bladder leiomyosarcoma has always been considered a highly aggressive tumour. Most patients are already in the advanced pathologic stage when the tumour is detected; less than $15 \%$ of tumours are identified in the T1 stage. $^{6}$ Metastatic signs of the disease usually portend a dismal prognosis. Administration of neoadjuvant chemotherapy should be considered in cases of locally advanced or metastatic disease. Whether neoadjuvant chemotherapy is given or not, surgical resection remains the cornerstone of treatment, with the surgical margin status being a strong predictor of outcome. ${ }^{7}$ Conventionally, radical cystectomy with wide

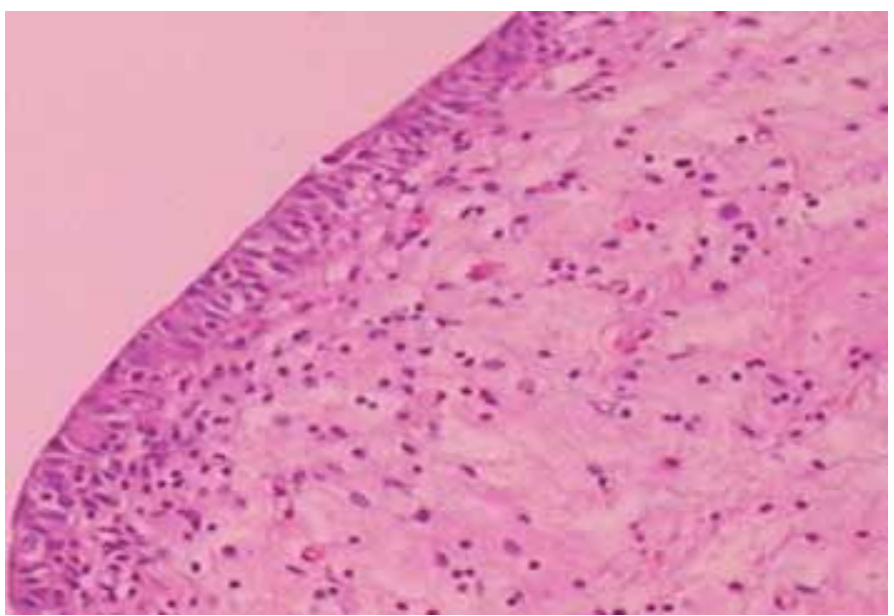

Fig. 4. Cystoscopic biopsy of bladder mucosa at postoperative 7 years, hematoxylin and eosin $\times 100$. 
margins is the gold standard in cases where surgical resection is possible. Although strict adherence to standard surgical technique (radical excision of the tumour with en bloc removal of the bladder to encompass the prostate and seminal vesicles in men and the uterus, cervix and vaginal cuff in women) results in low rates of positive surgical margins and low rates of local tumour recurrence, the loss of bladder function and large surgical trauma significantly decreases the quality of postoperative life. ${ }^{3}$ Transurethral resection of bladder leiomyosarcoma, in combination with chemoradiotherapy or laser photocoagulation, could be performed in individual cases (submucosal small tumours and/or inoperable patients), but it does not offer better long-term results than surgical resection. ${ }^{8}$

Partial surgery is now considered a reliable option to treat various tumours due to its function preservation and decreased aggressiveness. Recently, more cases of young patients with small tumours have been treated with partial cystectomy. Labanaris and colleagues reported a young female with a $1.2-\mathrm{cm}$ tumour who was treated with partial surgery with negative surgical margins. ${ }^{6}$ The 8 -month follow-up revealed no local recurrence or distant metastasis. Tsujita and colleagues reported a huge bladder diverticulum leiomyosarcoma $(>6 \mathrm{~cm})$ with mesentery infiltration in a 68-year-old man who underwent partial cystectomy. ${ }^{9}$ Unfortunately, the patient died of local recurrence and pulmonary metastasis 39 days after the operation. Strander and colleagues reported that adjuvant radiotherapy in combination with partial cystectomy was able to improve the prognosis of bladder leiomyosarcoma. ${ }^{10}$ Cumplido and colleagues reported a bladder leiomyosarcoma in a 75-yearold man who was treated with partial surgery and adjuvant chemoradiotherapy. ${ }^{11}$ The patient was tumour-free and living uneventfully at the time of this report.

This patient in our report underwent partial cystectomy only, with no adjuvant chemoradiotherapy. Fortunately, no tumour metastasis or recurrence was observed during the 7-year close follow-up period. An overview of the above-mentioned reports strongly suggests that a thorough staging evaluation of bladder leiomyosarcoma should be performed after admission. T1 or T2, MSKCC stage 1 or 2 small masses $(<4 \mathrm{~cm})$ that are not located in the neck and trigone of bladder may be indications for partial cystectomy of bladder leiomyosarcoma. Besides being beneficial, adjuvant chemoradiotherapy is not essential immediately after partial cystectomy. ${ }^{12}$ The overall local recurrence of bladder leiomyosarcoma is about $16 \%$, mostly recurring in the pelvis. The overall recurrence of distant metastases is about $53 \%$, the most common sites are the lungs, liver, bone and brain. ${ }^{7}$ Therefore, a close surveillance with abdominopelvic $C T$, chest $X$-ray and cystoscopy should be performed, especially in the first year postoperatively, which may be conducive to the early diagnosis of tumour recurrence and metastasis. Local recurrences should be treated with systemic chemotherapy and/or pelvic external beam radiotherapy. Multimodal and individual treatment is preferable in patients who present with distant metastasis, and chemotherapeutic agent doxorubicin and ifosfamide are known to have the greatest response. ${ }^{7}$ More recent studies suggest that bladder leiomyosarcoma may have a better prognosis than once believed, with a remarkable 5-year disease-specific survival rate of more than $50 \%{ }^{6}$

\section{Conclusion}

Early detection of bladder leiomyosarcoma and early intervention with an effective therapy significantly improve therapeutic efficacy. Partial cystectomy, as opposed to radical cystectomy, may be a reliable option for small bladder leiomyosarcoma $(<4 \mathrm{~cm})$ in a low MSKCC stage, because it may offer a similar therapeutic efficacy and a better quality of life.

Department of Urology, Shanghai Tenth People's Hospital of Tongii University, Shanghai, P.R. China

Competing interests: None declared.

This paper has been peer-reviewed.

\section{References}

1. Dahm P, Gschwend JE. Malignant non-urothelial neoplasms of the urinary bladder: a review. Eur Urol 2003;44:672-81.

2. Bergman RT, Kugel Al. Leiomyosarcoma of the urinary bladder. Urol Cutaneous Rev 1950;54:65-7.

3. Martin SA, Sears DL, Sebo TJ, et al. Smooth muscle neoplasms of the urinary bladder: a clinicopathologic comparison of leiomyoma and leiomyosarcoma. Am I Surg Pathol 2002;26:292-300.

4. Parekh DJ, Jung $C, O^{\prime}$ Conner J, et al. Leiomyosarcoma in urinary bladder after cyclophosphamide therapy for retinoblastoma and review of bladder sarcomas. Urology 2002;60:164-6.

5. Gronau S, Menz CK, Melzner I, et al. Immunohistomorphologic and molecular cytogenetic analysis of a carcinosarcoma of the urinary bladder. Virchows Arch 2002;440:436-40.

6. Labanaris AP, Zugor V, Meyer B, et al. Urinary bladder leiomyosarcoma in adults. Int Urol Nephrol 2008:40:311-6.

7. Spiess PE, Kassouf W, Steinberg JR, et al. Review of the M.D. Anderson experience in the treatment of bladder sarcoma. Urol Oncol 2007;25:38-45.

8. De Berardinis E, Giulianelli R, Zarrelli $G$, et al. Leiomyosarcoma of urinary bladder: personal experience in 3 cases over a 10-year period. Arch It Urol 1997;69(Suppl 1):73-80.

9. Tsujita Y, Sumitomo M, Tasaki S, et al. A case of leiomyosarcoma in a huge bladder diverticulum. Hinyokika Kiyo 2009;55:761-4.

10. Strander H, Turesson I, Cavallin-Stahl E. A systematic overview of radiation therapy effects in soft tissue sarcomas. Acta Oncol 2003;42:516-31.

11. Cumplido JD, Toral Peña JC. Bladder leiomyosarcoma. Partial cystectomy and adjuvant treatment. Arch Esp Urol 2009;62:320-2.

12. O'Sullivan B, Ward I, Catton C. Recent advances in radiotherapy for soft tissue sarcoma. Curr Oncol Rep 2003;5:274-81.

Correspondence: Dr. Jun-hua Zheng, Professor, Department of Urology, Shanghai Tenth People's Hospital of Tongii University, Shanghai 200072, P.R. China; fax: 86-21-65301051; zhengih0471@ sina.com 